\title{
New techniques for exact and approximate dynamic closest-point problems
}

\author{
Sanjiv Kapoor* Michiel Smid ${ }^{\dagger}$
}

August 22, 1995

\begin{abstract}
Let $S$ be a set of $n$ points in $\mathbb{R}^{D}$. It is shown that a range tree can be used to find an $L_{\infty}$-nearest neighbor in $S$ of any query point, in $O\left((\log n)^{D-1} \log \log n\right)$ time. This data structure has size $O\left(n(\log n)^{D-1}\right)$ and an amortized update time of $O\left((\log n)^{D-1} \log \log n\right)$. This result is used to solve the $(1+\epsilon)$-approximate $L_{2}$-nearest neighbor problem within the same bounds (up to a constant factor that depends on $\epsilon$ and $D$ ). In this problem, for any query point $p$, a point $q \in S$ is computed such that the euclidean distance between $p$ and $q$ is at most $(1+\epsilon)$ times the euclidean distance between $p$ and its true nearest neighbor. This is the first dynamic data structure for this problem having close to linear size and polylogarithmic query and update times.

New dynamic data structures are given that maintain a closest pair of $S$. For $D \geq 3$, a structure of size $O(n)$ is presented with amortized update time $O\left((\log n)^{D-1} \log \log n\right)$. The constant factor in this space (resp. time bound) is of the form $O(D)^{D}$ (resp. $\left.2^{O\left(D^{2}\right)}\right)$. For $D=2$ and any non-negative integer constant $k$, structures of size $O\left(n \log n /(\log \log n)^{k}\right)$ (resp. $\left.O(n)\right)$ are presented having an amortized update time of $O(\log n \log \log n)\left(\right.$ resp. $\left.O\left((\log n)^{2} /(\log \log n)^{k}\right)\right)$. Previously, no deterministic linear size data structure having polylogarithmic update time was known for this problem.
\end{abstract}

*Department of Computer Science, Indian Institute of Technology, Hauz Khas, New Delhi 110016, India. E-mail: skapoor@cse.iitd.ernet.in. The research on this work started when this author visited the Max-Planck-Institut für Informatik.

${ }^{\dagger}$ Max-Planck-Institut für Informatik, Im Stadtwald, D-66123 Saarbrücken, Germany. E-mail: michiel@mpi-sb.mpg.de. This author was supported by the ESPRIT Basic Research Actions Program, under contract No. 7141 (project ALCOM II). 


\section{Introduction}

Closest-point problems are among the basic problems in computational geometry. In such problems, a set $S$ of $n$ points in $D$-dimensional space is given and we have to store it in a data structure such that a point in $S$ nearest to a query point can be computed efficiently, or we have to compute a closest pair in $S$, or for each point in $S$ another point in $S$ that is closest to it. These problems are known as the nearest-neighbor problem, the closest pair problem, and the all-nearest-neighbors-problem, respectively. In the dynamic version of these problems, the set $S$ is changed by insertions and deletions of points.

It is assumed that the dimension $D \geq 2$ is a constant independent of $n$. Moreover, distances are measured in the $L_{t}$-metric, where $1 \leq t \leq \infty$ is a fixed real number. In this metric, the distance $d_{t}(p, q)$ between the points $p$ and $q$ is defined as $d_{t}(p, q)=\left(\sum_{i=1}^{D}\left|p_{i}-q_{i}\right|^{t}\right)^{1 / t}$ if $1 \leq t<\infty$, and for $t=\infty$ it is defined as $d_{\infty}(p, q)=\max _{1 \leq i \leq D}\left|p_{i}-q_{i}\right|$.

The planar version of the nearest-neighbor problem can be solved optimally, i.e., with $O(\log n)$ query time using $O(n)$ space, by means of Voronoi diagrams. (See [15].) In higher dimensions, however, the situation is much worse. The best results known are due to Clarkson [7] and Arya et al.[1]. In [7], a randomized data structure is given that finds a nearest-neighbor of a query point in $O(\log n)$ expected time. This structure has size $O\left(n^{\lceil D / 2\rceil+\delta}\right)$, where $\delta$ is an arbitrarily small positive constant. In [1], the problem is solved with an expected query time of $O\left(n^{1-1 /\lceil(D+1) / 2\rceil}(\log n)^{O(1)}\right)$ using $O(n \log \log n)$ space.

It seems that in higher dimensions it is impossible to obtain polylogarithmic query time using $O\left(n(\log n)^{O(1)}\right)$ space. Moreover, even in the planar case there is no dynamic data structure known that has polylogarithmic query and update times and that uses $O\left(n(\log n)^{O(1)}\right)$ space.

Therefore, it is natural to ask whether the approximate nearest-neighbor problem allows more efficient solutions. Let $\epsilon>0$. A point $q \in S$ is called a $(1+\epsilon)$-approximate neighbor of a point $p \in \mathbb{R}^{D}$, if $d_{t}(p, q) \leq(1+\epsilon) d_{t}\left(p, p^{*}\right)$, where $p^{*} \in S$ is the true nearest-neighbor of $p$.

This approximate neighbor problem was considered in Bern [5] and Arya et al.[1, 2]. In the latter paper, the problem is solved optimally: They give a deterministic data structure of size $O(n)$ that can find a $(1+\epsilon)$-approximate neighbor in $O(\log n)$ time, for any positive constant $\epsilon$. At this moment, however, no dynamic data structures are known for this problem.

In this paper, we first show that for the $L_{\infty}$-metric, the nearest-neighbor problem can be solved efficiently. To be more precise, we show that the range tree (see [12, $14,15,25])$ can be used to solve this problem. As a result, we solve the $L_{\infty}$-neighbor problem with a query time of $O\left((\log n)^{D-1} \log \log n\right)$ and an amortized update time of $O\left((\log n)^{D-1} \log \log n\right)$, using $O\left(n(\log n)^{D-1}\right)$ space. For the static version of this problem, the query time is $O\left((\log n)^{D-1}\right)$ and the space bound is $O\left(n(\log n)^{D-2}\right)$.

Using this result, we give a data structure that solves the approximate $L_{2}$-neighbor problem, for any positive constant $\epsilon$, within the same complexity bounds. (The constant factors depend on $D$ and $\epsilon$.) 


\begin{tabular}{|c||c|c|c|c|c|}
\hline mode & dimension & update time & w/a & space & reference \\
\hline \hline insertions & $D \geq 2$ & $\log n$ & w & $n$ & {$[17,18]$} \\
\hline deletions & $D \geq 2$ & $(\log n)^{D}$ & a & $n(\log n)^{D-1}$ & {$[23]$} \\
\hline fully dynamic & $D \geq 2$ & $\sqrt{n} \log n$ & w & $n$ & {$[16,20]$} \\
\hline fully dynamic & $D \geq 2$ & $(\log n)^{D} \log \log n$ & $\mathrm{a}$ & $n(\log n)^{D}$ & {$[22]$} \\
\hline fully dynamic & $D \geq 3$ & $(\log n)^{D-1} \log \log n$ & $\mathrm{a}$ & $n$ & this paper \\
\hline fully dynamic & 2 & $\log n \log \log n$ & $\mathrm{a}$ & $n \log n /(\log \log n)^{k}$ & this paper \\
\hline fully dynamic & 2 & $(\log n)^{2} /(\log \log n)^{k}$ & $\mathrm{a}$ & $n$ & this paper \\
\hline
\end{tabular}

Table 1: Deterministic data structures for the dynamic closest pair problem. In the last two lines, $k$ is an arbitrary non-negative integer constant. All bounds are "big-oh" with constant factors that depend on the dimension $D$ and, in the last two lines, on $D$ and $k$. The update times are either worst-case (w) or amortized (a).

We also consider the dynamic closest pair problem. Note that the static version has been solved already for a long time. Several algorithms are known that compute a closest pair in $O(n \log n)$ time, which is optimal. (See $[4,10,15,19]$.) The dynamic version, however, has been investigated only recently. In Table 1, we give an overview of the currently best known data structures for maintaining a closest pair under insertions and/or deletions of points. For an up-to-date survey, we refer the reader to Schwarz's Ph.D. Thesis [17].

Note that all data structures of Table 1 are deterministic and can be implemented in the algebraic computation tree model. If we add randomization to this model, then there is a data structure of size $O(n)$ that maintains a closest pair in $O\left((\log n)^{2}\right)$ expected time per update. (See Golin et al.[9].)

In this paper, we give new deterministic data structures for the dynamic closest pair problem. These structures are based on our solution to the $L_{\infty}$-neighbor problem, data structures for maintaining boxes of constant overlap, and a new transformation. Given any dynamic closest pair data structure having more than linear size, this transformation produces another dynamic closest pair structure that uses less space. The complexity bounds of the new structures are shown in the last three lines of Table 1. The results of the last two lines are obtained by applying the transformation repeatedly. Note that we obtain the first linear size deterministic data structure that maintains a closest pair in polylogarithmic time.

Finally, we consider the all-nearest-neighbors problem. Again, in the planar case, the problem can be solved using Voronoi diagrams. For the $D$-dimensional case, Vaidya [24] has given an optimal $O(n \log n)$ time algorithm that computes the $L_{t^{-}}$ neighbor for each point of $S$. No non-trivial solutions seem to be known for maintaining for each point its nearest-neighbor. One of our data structures (not the ones mentioned in Table 1) for maintaining the closest pair basically maintains the $L_{\infty^{-}}$ neighbor of each point in $S$. As a result, we get a data structure of size $O\left(n(\log n)^{D-1}\right)$ that maintains for each point in $S$ its $L_{\infty}$-neighbor in $O\left((\log n)^{D-1} \log \log n\right)$ amortized time per update.

We want to remark here that all our algorithms use classical and well-understood data structures, such as range trees, segment trees, and skewer trees. Moreover, we 
apply the well known technique of fractional cascading $([6,14])$ several times.

The rest of this paper is organized as follows. In Section 2, we recall the definition of a range tree. This data structure is used in Section 3 to solve the $L_{\infty}$-neighbor problem. In Section 4, we give the data structure for solving the approximate $L_{2^{-}}$ nearest neighbor problem.

Our first data structure for maintaining the closest pair stores a dynamically changing set of boxes that are of constant overlap, i.e., there is a constant $c$ such that each box contains the centers of at most $c$ boxes in its interior. We have to maintain such boxes under insertions and deletions such that for any query point $p \in \mathbb{R}^{D}$, we can find all boxes that contain $p$. In Section 5 , we give two data structures for this problem. The first one is based on segment trees. (See [13, 15].) Therefore, its size is superlinear. We also give a linear size solution having a slightly worse update time in the planar case. This solution is based on skewer trees. (See $[8,21]$.)

In Section 6, we use the results obtained to give a new data structure that maintains the closest pair in a point set. This structure has size $O\left(n(\log n)^{D-1}\right)$ and an amortized update time of $O\left((\log n)^{D-1} \log \log n\right)$. It immediately gives the dynamic data structure for the all- $L_{\infty}$-nearest-neighbors problem. In Section 7 , we give the transformation that reduces the size of a dynamic closest pair structure. Applying this transformation repeatedly to the structure of Section 6 gives the new results mentioned in Table 1. Some concluding remarks are given in Section 8.

\section{Range trees}

In this section, we recall the definition of a range tree. See $[12,13,15,25]$. The coordinates of a point $p$ in $\mathbb{R}^{D}$ are denoted by $p_{i}, 1 \leq i \leq D$. Moreover, we denote by $p^{\prime}$ the point $\left(p_{2}, \ldots, p_{D}\right)$ in $\mathbb{R}^{D-1}$. If $S$ is a set of points in $\mathbb{R}^{D}$, then we define $S^{\prime}:=\left\{p^{\prime}: p \in S\right\}$. We note that $S^{\prime}$ is to be considered as a multiset, i.e., elements may occur more than once.

The range tree: Let $S$ be a set of $n$ points in $\mathbb{R}^{D}$. A $D$-dimensional range tree for the set $S$ is defined as follows. For $D=1$, it is a balanced binary search tree, storing the elements of $S$ in sorted order in its leaves.

For $D>1$, it consists of a balanced binary search tree, called the main tree, storing the points of $S$ in its leaves, sorted by their first coordinates. For each internal node $v$ of this main tree, let $S_{v}$ be the set of points that are stored in its subtree. Node $v$ contains a pointer to the rightmost leaf in its subtree and (a pointer to) an associated structure, which is a $(D-1)$-dimensional range tree for the set $S_{v}^{\prime}$.

Hence, a 2-dimensional range tree for a set $S$ consists of a binary tree, storing the points of $S$ in its leaves sorted by their $x$-coordinates. Each internal node $v$ of this tree contains a pointer to the rightmost leaf in its subtree and (a pointer to) a binary tree that stores the points of $S_{v}$ in its leaves sorted by their $y$-coordinates.

Let $p$ be any point in $\mathbb{R}^{D}$. Consider the set $\left\{r \in S: r_{1} \geq p_{1}\right\}$, i.e., the set of all points in $S$ having a first coordinate that is at least equal to $p$ 's first coordinate. Using the range tree, we can decompose this set into $O(\log n)$ canonical subsets: 
Initialize $M:=\emptyset$. Starting in the root of the main tree, search for the leftmost leaf storing a point whose first coordinate is at least equal to $p_{1}$. During this search, each time we move from a node $v$ to its left son, add the right son of $v$ to the set $M$. Let $v$ be the leaf in which this search ends. If the point stored in this leaf has a first coordinate that is at least equal to $p_{1}$, then add $v$ to the set $M$.

Lemma 1 The set $M$ of nodes of the main tree that is computed by the given algorithm satisfies

$$
\left\{r \in S: r_{1} \geq p_{1}\right\}=\bigcup_{v \in M} S_{v} .
$$

Moreover, $M$ is computed in $O(\log n)$ time.

Range trees can be maintained under insertions and deletions of points such that each binary tree that is part of the structure has a height logarithmic in the number of its leaves. The update algorithms use dynamic fractional cascading. For details, we refer the reader to Mehlhorn and Näher [14]. In the next theorem, we state the complexity of the range tree.

Theorem 1 A D-dimensional range tree for a set of $n$ points has $O\left(n(\log n)^{D-1}\right)$ size and can be maintained in $O\left((\log n)^{D-1} \log \log n\right)$ amortized time per insertion and deletion.

\section{The $L_{\infty}$-neighbor problem}

Recall the following notations. For any point $p=\left(p_{1}, p_{2}, \ldots, p_{D}\right) \in \mathbb{R}^{D}$, we denote by $p^{\prime}$ the point $\left(p_{2}, \ldots, p_{D}\right)$ in $\mathbb{R}^{D-1}$. If $S$ is a set of points in $\mathbb{R}^{D}$, then $S^{\prime}=\left\{p^{\prime}: p \in S\right\}$. Finally, if $v$ is a node of the main tree of a range tree storing a set $S$, then $S_{v}$ denotes the set of points that are stored in the subtree of $v$.

Let $S$ be a set of $n$ points in $\mathbb{R}^{D}$. We want to store this set into a data structure such that for any query point $p \in \mathbb{R}^{D}$, we can find a point in $S$ having minimal $L_{\infty}$-distance to $p$. Such a point is called an $L_{\infty}$-neighbor of $p$ in $S$.

Let $q$ be an $L_{\infty}$-neighbor of $p$ in the set $\left\{s \in S: s_{1} \geq p_{1}\right\}$. We call $q$ a right- $L_{\infty^{-}}$ neighbor of $p$ in $S$. Similarly, a point $r$ is called a left- $L_{\infty}$-neighbor of $p$ in $S$, if it is an $L_{\infty}$-neighbor of $p$ in the set $\left\{s \in S: s_{1} \leq p_{1}\right\}$. In order to guarantee that both neighbors always exist, we add the $2 D$ artificial points $\left(a_{1}, \ldots, a_{D}\right)$, where all $a_{i}$ are zero, except for one which is either $\infty$ or $-\infty$, to the set $S$. Note that none of these points can be $L_{\infty}$-neighbor of any query point.

The data structure that solves the $L_{\infty}$-neighbor problem is just a $D$-dimensional range tree storing the points of $S$ and the artificial points. In Figure 1, our recursive algorithm is given that finds an $L_{\infty}$-neighbor of any query point in $\mathbb{R}^{D}$.

We prove the correctness of this query algorithm. It is clear that the algorithm is correct in the 1-dimensional case. So let $D \geq 2$ and assume that the algorithm is correct for smaller values of $D$. The following lemma turns out to be useful.

Lemma 2 Let $p \in \mathbb{R}^{D}$ and let $q$ be its right- $L_{\infty}$-neighbor in $S$. Let $w$ be any node in the main tree of the range tree such that $q \in S_{w}$ and $p$ has a first coordinate that is at 
Algorithm Neighbor $(p, S, D) \quad\left(*\right.$ returns an $L_{\infty}$-neighbor of $p$ in $\left.S^{*}\right)$

begin

1. Assume $D=1$.

$q^{L}:=$ maximal element in the 1-dimensional range tree that is less than $p$;

$q^{R}:=$ minimal element in the 1-dimensional range tree that is at least equal to $p$;

if $\left|p-q^{R}\right| \leq\left|p-q^{L}\right|$ then return $q^{R}$ else return $q^{L}$ fi;

2. Assume $D \geq 2$.

2a. Compute a right- $L_{\infty}$-neighbor:

Stage 1. Compute the set $M$ of nodes of the main tree, see Lemma 1.

Number these nodes $v_{1}, v_{2}, \ldots, v_{m}, m=|M|$, where $v_{i}$ is closer to the root than $v_{i-1}, 2 \leq i \leq m$.

Stage 2. (* one of the sets $S_{v_{i}}$ contains a right- $L_{\infty}$-neighbor of $p^{*}$ )

$C:=\emptyset ; i:=1 ;$ stop $:=$ false;

while $i \leq m$ and stop $=$ false

do $x^{\prime}:=\operatorname{Neighbor}\left(p^{\prime}, S_{v_{i}}^{\prime}, D-1\right)$;

$r:=$ the point stored in the rightmost leaf of the subtree of $v_{i}$;

if $d_{\infty}\left(p^{\prime}, x^{\prime}\right)>\left|p_{1}-r_{1}\right|$

then $C:=C \cup\{x\} ; i:=i+1$

else $v:=v_{i} ;$ stop $:=$ true

fi

od;

if $s t o p=$ false

then $q^{R}:=$ a point of $C$ having minimal $L_{\infty}$-distance to $p$; goto $\mathbf{2 b}$

fi;

Stage 3. (* the set $C \cup S_{v}$ contains a right- $L_{\infty}$-neighbor of $p^{*}$ )

while $v$ is not a leaf

do $w:=$ left son of $v$;

$x^{\prime}:=\operatorname{Neighbor}\left(p^{\prime}, S_{w}^{\prime}, D-1\right) ;$

$r:=$ the point stored in the rightmost leaf of the subtree of $w$;

if $d_{\infty}\left(p^{\prime}, x^{\prime}\right)>\left|p_{1}-r_{1}\right|$

then $C:=C \cup\{x\} ; v:=$ right son of $v$

else $v:=w$

fi

od;

$q^{R}:=$ a point of $C \cup S_{v}$ having minimal $L_{\infty}$-distance to $p$;

2b. Compute a left- $L_{\infty}$-neighbor:

In a completely symmetric way, compute a left- $L_{\infty}$-neighbor $q^{L}$ of $p$;

2c. if $d_{\infty}\left(p, q^{R}\right) \leq d_{\infty}\left(p, q^{L}\right)$ then return $q^{R}$ else return $q^{L} \mathbf{f i}$

end

Figure 1: Finding an $L_{\infty}$-neighbor. 
most equal to the first coordinate of any point in $S_{w}$. Let $r$ be the point of $S$ that is stored in the rightmost leaf of w's subtree. Finally, let

$$
N:=\left|\left\{x \in S_{w}:\left|p_{j}-x_{j}\right| \leq\left|p_{1}-r_{1}\right|, 2 \leq j \leq D\right\}\right| .
$$

If $N=0$, then $q^{\prime}$ is an $L_{\infty}$-neighbor of $p^{\prime}$ in the $(D-1)$-dimensional set $S_{w}^{\prime}$.

Proof: The proof is by contradiction. So let $s$ be a point in $S_{w}$ such that $s^{\prime}$ is an $L_{\infty^{-}}$ neighbor of $p^{\prime}$ in the set $S_{w}^{\prime}$ and assume that $d_{\infty}\left(p^{\prime}, s^{\prime}\right)<d_{\infty}\left(p^{\prime}, q^{\prime}\right)$. Let $\delta:=\left|p_{1}-r_{1}\right|$.

Since $q$ and $s$ are elements of $S_{w}$, we have $\left|p_{1}-q_{1}\right| \leq \delta$ and $\left|p_{1}-s_{1}\right| \leq \delta$. Since $N=0$, there is a $j, 2 \leq j \leq D$, such that $\left|p_{j}-q_{j}\right|>\delta$. Therefore, $d_{\infty}(p, q)=d_{\infty}\left(p^{\prime}, q^{\prime}\right)$. Similarly, there is a $k, 2 \leq k \leq D$, such that $\left|p_{k}-s_{k}\right|>\delta$ and, hence, $d_{\infty}(p, s)=$ $d_{\infty}\left(p^{\prime}, s^{\prime}\right)$. This implies that $d_{\infty}(p, s)<d_{\infty}(p, q)$, i.e., $q$ is not a right- $L_{\infty}$-neighbor of $p$ in $S$. This is a contradiction.

We analyze part $\mathbf{2 a}$ of the algorithm. As we will see, this part computes a right$L_{\infty}$-neighbor of $p$. Consider the nodes $v_{1}, \ldots, v_{m}$ that are computed in Stage 1 . We know that

$$
\left\{r \in S: r_{1} \geq p_{1}\right\}=\bigcup_{i=1}^{m} S_{v_{i}} .
$$

Hence, one of the sets $S_{v_{i}}$ contains a right- $L_{\infty}$-neighbor of $p$.

Note that each point in $S_{v_{i-1}}$ has a first coordinate that is at most equal to the first coordinate of any point in $S_{v_{i}}, 2 \leq i \leq m$.

Lemma 3 If the variable stop has value false after the while-loop of Stage 2 has been completed, then the set $C$ contains a right- $L_{\infty}$-neighbor of $p$ in $S$.

Proof: Let $i, 1 \leq i \leq m$, be an index such that the set $S_{v_{i}}$ contains a right- $L_{\infty^{-}}$ neighbor $q$ of $p$. We consider what happens during the $i$-th iteration of the while-loop. Let $x$ and $r$ be the points that are selected in this iteration. Since $d_{\infty}\left(p^{\prime}, x^{\prime}\right)>\left|p_{1}-r_{1}\right|$, it is clear that the value of the integer

$$
N:=\left|\left\{y \in S_{v_{i}}:\left|p_{j}-y_{j}\right| \leq\left|p_{1}-r_{1}\right|, 2 \leq j \leq D\right\}\right|
$$

is zero. Then, Lemma 2 implies that $q^{\prime}$ is an $L_{\infty}$-neighbor of $p^{\prime}$ in $S_{v_{i}}^{\prime}$. Hence, $d_{\infty}\left(p^{\prime}, x^{\prime}\right)=d_{\infty}\left(p^{\prime}, q^{\prime}\right)$. Since $d_{\infty}\left(p^{\prime}, x^{\prime}\right)=d_{\infty}(p, x)$ and $d_{\infty}\left(p^{\prime}, q^{\prime}\right)=d_{\infty}(p, q)$, it follows that $d_{\infty}(p, x)=d_{\infty}(p, q)$. This proves that $x$ is a right- $L_{\infty}$-neighbor of $p$. Since $x$ is added to $C$ during this iteration, the proof is completed.

Lemma 4 If the variable stop has value true after the while-loop of Stage 2 has been completed, then the set $C \cup S_{v}$ contains a right- $L_{\infty}$-neighbor of $p$ in $S$.

Proof: Consider the integer $i$ such that during the $i$-th iteration of the while-loop the variable stop is set to the value true. Then $v=v_{i}$.

We distinguish two cases. First let $j \geq i+1$ and assume that the set $S_{v_{j}}$ contains a right- $L_{\infty}$-neighbor $q$ of $p$ in $S$. Consider what happens during the $i$-th iteration of the while-loop. Let $x$ and $r$ be the points that are selected during this iteration. Moreover, 
let $\delta:=\left|p_{1}-r_{1}\right|$. Since $\left|p_{1}-x_{1}\right| \leq \delta$ and $d_{\infty}\left(p^{\prime}, x^{\prime}\right) \leq \delta$, we have $d_{\infty}(p, x) \leq \delta$. Since $q \in S_{v_{j}}$, we have

$$
d_{\infty}(p, q) \geq q_{1}-p_{1} \geq r_{1}-p_{1}=\delta
$$

This implies that $d_{\infty}(p, x) \leq d_{\infty}(p, q)$. Hence, $x$ is also a right- $L_{\infty}$-neighbor of $p$. Since $x \in S_{v}$, the claim of the lemma follows.

Next let $1 \leq j \leq i$ and assume that the set $S_{v_{j}}$ contains a right- $L_{\infty}$-neighbor of $p$ in $S$. If $j=i$, then we are done. If $j<i$, then in the same way as in the proof of Lemma 3 , it follows that such a neighbor is added to $C$ during the $j$-th iteration of the while-loop. This completes the proof.

Lemma 5 During the while-loop of Stage 3, the set $C \cup S_{v}$ contains a right- $L_{\infty}$ neighbor of $p$ in $S$.

Proof: The previous lemma implies that the claim holds before the while-loop is entered. Consider an iteration and assume that $C \cup S_{v}$ contains a right- $L_{\infty}$-neighbor of $p$. (We consider $C$ as it is at the beginning of this iteration.) Let $x$ and $r$ be the points that are selected during this iteration. Moreover, let $w_{l}$ (resp. $w_{r}$ ) be the left (resp. right) son of $v$.

First assume that $d_{\infty}\left(p^{\prime}, x^{\prime}\right)>\left|p_{1}-r_{1}\right|$. We have to show that $C \cup\{x\} \cup S_{w_{r}}$ contains a right- $L_{\infty}$-neighbor of $p$. Since the set $C \cup S_{w_{l}} \cup S_{w_{r}}$ contains a right- $L_{\infty}$-neighbor of $p$, we only have to consider the case where the set $S_{w_{l}}$ contains such a neighbor. In this case, it follows in the same way as in the proof of Lemma 3 that point $x$ is a right- $L_{\infty}$-neighbor of $p$. Since this point is added to $C$ during this iteration, the claim follows.

It remains to consider the case where $d_{\infty}\left(p^{\prime}, x^{\prime}\right) \leq\left|p_{1}-r_{1}\right|$. Now we have to show that the set $C \cup S_{w_{l}}$ contains a right- $L_{\infty}$-neighbor of $p$. Assume that $S_{w_{r}}$ contains such a neighbor. As in the proof of the previous lemma, it follows that $x$ is also a right- $L_{\infty}$-neighbor of $p$. But, $x$ is an element of $S_{w_{l}}$. This completes the proof.

Lemmas 3 and 5 imply that point $q^{R}$ which is computed in part $\mathbf{2 a}$ is a right- $L_{\infty^{-}}$ neighbor of $p$ in $S$. Similarly, point $q^{L}$ computed in part $\mathbf{2} \mathbf{b}$ is a left- $L_{\infty}$-neighbor of $p$. This proves that the point that is returned in part $\mathbf{2 c}$ is an $L_{\infty}$-neighbor of $p$. Hence, algorithm Neighbor $(p, S, D)$ is correct.

Lemma 6 For $D \geq 2$, the running time of algorithm Neighbor $(p, S, D)$ is bounded by $O\left((\log n)^{D-1}\right)$.

Proof: Let $Q(n, D)$ denote the running time on a set of $n$ points in $\mathbb{R}^{D}$. It is clear that $Q(n, 1)=O(\log n)$. Let $D \geq 2$. Consider part 2a. Stage 1 takes $O(\log n)$ time. The while-loop in Stage 2 takes time $O(Q(n, D-1) \log n)$. If stop $=$ false after this loop, then $O(|C|)=O(\log n)$ time is needed to select the point $q^{R}$. Hence, Stage 2 takes time $O(Q(n, D-1) \log n)$. Stage 3 takes the same amount of time. Part $\mathbf{2} \mathbf{b}$ can be analyzed in the same way. Clearly, part $\mathbf{2 c}$ takes only constant time. This proves that $Q(n, D)=O(Q(n, D-1) \log n)$, implying that $Q(n, D)=O\left((\log n)^{D}\right)$.

Now consider the planar case. The algorithm follows a path in the main tree and locates the $y$-coordinate of the query point in the associated structure - a binary search 
tree - of some of the nodes on this path. It is well known that layering or fractional cascading (see $[6,15])$ can be applied to improve the query time from $O\left((\log n)^{2}\right)$ to $O(\log n)$, i.e., $Q(n, 2)=O(\log n)$. As a result, the query time for the $D$-dimensional case, where $D \geq 2$, is improved to $O\left((\log n)^{D-1}\right)$.

Hence, we have a data structure for the $L_{\infty}$-neighbor problem that has a query time of $O\left((\log n)^{D-1}\right)$ and that uses $O\left(n(\log n)^{D-1}\right)$ space. We can improve the space bound by noting that the planar version can be solved by means of the $L_{\infty}$-Voronoi diagram. (See Lee [11].) That is, the planar $L_{\infty}$-neighbor problem has a solution with a query time of $O(\log n)$ using only $O(n)$ space. As a result, the $D$-dimensional problem can be solved with a query time of $O\left((\log n)^{D-1}\right)$ using $O\left(n(\log n)^{D-2}\right)$ space.

Until now, we only considered the static version of the problem. We saw in Theorem 1 that range trees can be maintained under insertions and deletions of points. Therefore, our solution that is based on the range tree only can be used for the dynamic problem. Because of dynamic fractional cascading, the query time increases by a factor of $O(\log \log n)$. This proves the following result.

Theorem 2 Let $S$ be a set of $n$ points in $\mathbb{R}^{D}$. There exists a data structure of size $O\left(n(\log n)^{D-2}\right)$ that, given a query point $p \in \mathbb{R}^{D}$, finds an $L_{\infty}$-neighbor of $p$ in $S$ in $O\left((\log n)^{D-1}\right)$ time.

For the dynamic version of the problem, there is a structure of size $O\left(n(\log n)^{D-1}\right)$ having $O\left((\log n)^{D-1} \log \log n\right)$ query time and $O\left((\log n)^{D-1} \log \log n\right)$ amortized update time.

Remark: Let $c$ be a positive integer. The range tree can also be used to compute the $c L_{\infty}$-neighbors of a query point. The algorithm is basically the same. If $c$ is a constant, the query time remains the same as in the above theorem.

\section{The approximate $L_{2}$-neighbor problem}

We mentioned in the introduction that the $L_{2}$-nearest-neighbor problem is hard to solve exactly. Therefore, it is natural to consider a weaker version of the problem.

Let $S$ be a set of $n$ points in $\mathbb{R}^{D}$ and let $\epsilon>0$ be a fixed constant. For any point $p \in \mathbb{R}^{D}$, we denote by $p^{*}$ its $L_{2}$-neighbor in $S$, i.e., $p^{*}$ is a point of $S$ such that $d_{2}\left(p, p^{*}\right)=\min \left\{d_{2}(p, q): q \in S\right\}$. A point $q \in S$ is called a $(1+\epsilon)$-approximate $L_{2}$-neighbor of $p$ if $d_{2}(p, q) \leq(1+\epsilon) d_{2}\left(p, p^{*}\right)$.

We want to store the set $S$ in a data structure such that for any query point $p \in \mathbb{R}^{D}$ we can find a $(1+\epsilon)$-approximate $L_{2}$-neighbor of it.

Let $p \in \mathbb{R}^{D}$ and let $q$ be an $L_{\infty}$-neighbor of $p$ in $S$. Let $\delta:=d_{\infty}\left(p, p^{*}\right)$ and consider the $D$-dimensional axes-parallel box centered at $p$ having sides of length $2 \delta$. Clearly, $q$ lies inside or on the boundary of this box. Therefore, $d_{2}(p, q) \leq \sqrt{D} \cdot \delta$. Since $\delta=d_{\infty}\left(p, p^{*}\right) \leq d_{2}\left(p, p^{*}\right)$, we infer that $d_{2}(p, q) \leq \sqrt{D} \cdot d_{2}\left(p, p^{*}\right)$, i.e., $q$ is a $\sqrt{D}$-approximate $L_{2}$-neighbor of $p$.

This shows that we can solve the $\sqrt{D}$-approximate $L_{2}$-neighbor problem using the results of the previous section. 
We extend this solution. First note that the $L_{\infty}$-metric depends on the coordinate system: If we rotate the $(X Y)$-system, then the $L_{\infty}$-metric changes. The $L_{2}$-metric, however, is invariant under such rotations. We store the set $S$ in a constant number of range trees, where each range tree stores the points according to its own coordinate system. Then given $p$, we use the range trees to compute $L_{\infty}$-neighbors in all coordinate systems. As we will see, one of these $L_{\infty}$-neighbors is a $(1+\epsilon)$-approximate $L_{2}$-neighbor of $p$.

Let $\left(\mathcal{F}_{i}\right)$ be a family of orthonormal coordinate systems all sharing the same origin. The coordinates of any point $x$ in the system $\mathcal{F}_{i}$ are denoted by $x_{i 1}, x_{i 2}, \ldots, x_{i D}$. Moreover, $d_{i, \infty}(\cdot, \cdot)$ denotes the $L_{\infty}$-distance function in $\mathcal{F}_{i}$. Assume that for any point $x$ in $D$-dimensional space there is an index $i$ such that for all $1 \leq j \leq D$,

$$
0 \leq x_{i j} \leq x_{i D} \leq(1+\epsilon) x_{i j}
$$

i.e., in $\mathcal{F}_{i}$ all coordinates of $x$ are non-negative and almost equal. Note that the family $\left(\mathcal{F}_{i}\right)$ is independent of the set $S$.

In Yao [26], it is shown how such a family consisting of $O\left((c / \epsilon)^{D-1}\right)$ coordinate systems can be constructed. (Here, $c$ is a constant.) In the planar case, such a family is easily obtained: Let $0<\phi<\pi / 4$ be such that $\tan \phi=\epsilon /(2+\epsilon)$. For $0 \leq i<2 \pi / \phi$, let $X_{i}$ (resp. $Y_{i}$ ) be the directed line that makes an angle of $i \cdot \phi$ with the positive $X$-axis (resp. $Y$-axis). Then, $\mathcal{F}_{i}$ is the $\left(X_{i} Y_{i}\right)$-coordinate system.

To prove that (1) holds for this family, let $x$ be any point in the plane and let $\gamma$ be the angle that the line segment $\vec{x}$ between the origin and $x$ makes with the positive $X$-axis.

First assume that $\pi / 4 \leq \gamma<2 \pi$. Let $i:=\lfloor(\gamma-\pi / 4) / \phi\rfloor$ and let $\gamma_{i}$ be the angle between $\vec{x}$ and the positive $X_{i}$-axis. Then $\gamma_{i}=\gamma-i \cdot \phi$ and this angle lies in between $\pi / 4$ and $\pi / 4+\phi$. Therefore, the coordinates $x_{i 1}$ and $x_{i 2}$ of $x$ in $\mathcal{F}_{i}$ satisfy

$$
x_{i 2} / x_{i 1}=\tan \gamma_{i} \geq \tan \pi / 4=1
$$

and

$$
x_{i 2} / x_{i 1}=\tan \gamma_{i} \leq \tan (\pi / 4+\phi)=\frac{1+\tan \phi}{1-\tan \phi}=1+\epsilon .
$$

If $0 \leq \gamma<\pi / 4$, then we take $i:=\lfloor(7 \pi / 4+\gamma) / \phi\rfloor$. In this case, $\gamma_{i}=2 \pi-i \cdot \phi+\gamma$ and this angle lies in between $\pi / 4$ and $\pi / 4+\phi$. This proves that $x_{i 2} / x_{1}$ lies in between 1 and $1+\epsilon$.

Hence, the family $\left(\mathcal{F}_{i}\right)$ satisfies the assumptions made in $(1)$. Moreover, the number of coordinate systems is at most

$$
1+\frac{2 \pi}{\phi}=1+\frac{2 \pi}{\arctan \epsilon /(2+\epsilon)}=O(1 / \epsilon)
$$

The data structure for approximate $L_{2}$-neighbor queries: For each index $i$, let $S_{i}$ denote the set of points in $S$ with coordinates in the system $\mathcal{F}_{i}$. The data structure consists of a collection of range trees; the $i$-th range tree stores the set $S_{i}$. 
Finding an approximate $L_{2}$-neighbor: Let $p \in \mathbb{R}^{D}$ be a query point. For each index $i$, use the $i$-th range tree to find an $L_{\infty}$-neighbor $q^{(i)}$ of $p$ in $S_{i}$. Report an $L_{\infty}$-neighbor that has minimal $L_{2}$-distance to $p$.

Lemma 7 The query algorithm reports a $(1+\epsilon)$-approximate $L_{2}$-neighbor of $p$.

Proof: Consider an exact $L_{2}$-neighbor $p^{*}$ of $p$. Let $i$ be an index such that the coordinates of $p^{*}-p$ in $\mathcal{F}_{i}$ satisfy

$$
0 \leq p_{i j}-p_{i j}^{*} \leq p_{i D}-p_{i D}^{*} \leq(1+\epsilon)\left(p_{i j}-p_{i j}^{*}\right), \quad 1 \leq j \leq D .
$$

Let $q$ be the point that is reported by the algorithm. Since $d_{2}(p, q) \leq d_{2}\left(p, q^{(i)}\right)$, it suffices to prove that $d_{2}\left(p, q^{(i)}\right) \leq(1+\epsilon) d_{2}\left(p, p^{*}\right)$.

Let $B$ be the $D$-dimensional box centered at $p$ with sides of length $2 d_{i, \infty}\left(p, p^{*}\right)$ that are parallel to the axes of $\mathcal{F}_{i}$. Since $q^{(i)}$ is an $L_{\infty}$-neighbor of $p$ in $S_{i}$, this point must lie inside or on the boundary of $B$. It follows that

$$
d_{2}\left(p, q^{(i)}\right) \leq \sqrt{D} \cdot d_{i, \infty}\left(p, p^{*}\right)
$$

Note that $d_{i, \infty}\left(p, p^{*}\right)=p_{i D}-p_{i D}^{*}$. Moreover,

$$
\left(d_{2}\left(p, p^{*}\right)\right)^{2}=\sum_{j=1}^{D}\left(p_{i j}-p_{i j}^{*}\right)^{2} \geq \sum_{j=1}^{D}\left(\frac{p_{i D}-p_{i D}^{*}}{1+\epsilon}\right)^{2}=\frac{D}{(1+\epsilon)^{2}}\left(d_{i, \infty}\left(p, p^{*}\right)\right)^{2} .
$$

Hence,

$$
d_{2}\left(p, q^{(i)}\right) \leq \sqrt{D} \cdot d_{i, \infty}\left(p, p^{*}\right) \leq \sqrt{D} \cdot \frac{1+\epsilon}{\sqrt{D}} \cdot d_{2}\left(p, p^{*}\right)=(1+\epsilon) d_{2}\left(p, p^{*}\right) .
$$

This proves the lemma.

Hence, we have solved the $(1+\epsilon)$-approximate $L_{2}$-neighbor problem. The complexity of our solution follows immediately from Theorem 2 . We have proved the following theorem.

Theorem 3 Let $S$ be a set of $n$ points in $\mathbb{R}^{D}$ and let $\epsilon$ be a positive constant. There exists a data structure of size $O\left(n(\log n)^{D-2}\right)$ that, given a query point $p \in \mathbb{R}^{D}$, finds a $(1+\epsilon)$-approximate $L_{2}$-neighbor of $p$ in $S$ in $O\left((\log n)^{D-1}\right)$ time.

For the dynamic version of the problem, there is a data structure with a query time of $O\left((\log n)^{D-1} \log \log n\right)$ and an amortized update time of $O\left((\log n)^{D-1} \log \log n\right)$ that uses space $O\left(n(\log n)^{D-1}\right)$.

In all complexity bounds, the constant factor is proportional to $(c / \epsilon)^{D-1}$ for some fixed $c$. 


\section{The containment problem for boxes of constant overlap}

A box is a $D$-dimensional axes-parallel cube, i.e., it is of the form

$$
\left[a_{1}: a_{1}+\delta\right] \times\left[a_{2}: a_{2}+\delta\right] \times \ldots \times\left[a_{D}: a_{D}+\delta\right]
$$

for real numbers $a_{1}, a_{2}, \ldots, a_{D}$ and $\delta>0$. The center of this box is the point $\left(a_{1}+\right.$ $\left.\delta / 2, a_{2}+\delta / 2, \ldots, a_{D}+\delta / 2\right)$.

Let $S$ be a set of $n$ boxes in $\mathbb{R}^{D}$ that are of constant overlap, i.e., there is an integer constant $c_{D}$-possibly depending on the dimension - such that each box $B$ of $S$ contains the centers of at most $c_{D}$ boxes in its interior. (Here, we also count the center of $B$ itself. Note that many boxes may have their centers on the boundary of $B$.)

We want to store these boxes in a data structure such that for any query point $p \in \mathbb{R}^{D}$, we can find all boxes that contain $p$. Note that we distinguish between "being contained in a box" and "being contained in the interior of a box". The following lemma shows that a point $p$ can be contained in only a constant number of boxes.

Lemma 8 Any point $p \in \mathbb{R}^{D}$ is contained in at most $2^{1+D^{2}} c_{D}$ boxes of $S$.

Proof: The proof is by induction on $D$. So let $D=1$. Assume w.l.o.g. that $p=0$. Let $S_{+}$be the set of all boxes of $S$ whose centers are positive. Let $b_{1}, b_{2}, \ldots, b_{m}$ be the centers of the boxes in $S_{+}$that contain $p$. Assume w.l.o.g. that $b_{1}=\max \left\{b_{i}: 1 \leq i \leq\right.$ $m\}$. The interior of the box having $b_{1}$ as its center contains all centers $b_{i}, 1 \leq i \leq m$. Hence, the constant overlap property implies that $m \leq c_{1}$, i.e., point $p$ is contained in at most $c_{1}$ boxes of $S_{+}$.

By a symmetric argument, point $p$ is contained in at most $c_{1}$ boxes of $S$ whose centers are negative. It remains to consider the boxes having $p$ as their centers. The constant overlap property directly implies that there are at most $c_{1}$ such boxes.

This proves that there are at most $3 c_{1}$ boxes in the entire set $S$ that contain $p$.

Now let $D \geq 2$ and assume the lemma holds for dimension $D-1$. Again, we assume w.l.o.g. that $p$ is the origin. Let $S_{+}$be the set of all boxes of $S$ whose centers have positive coordinates. We will show that there are at most $c_{D}$ boxes in $S_{+}$that contain $p$.

Let $b_{1}, b_{2}, \ldots, b_{m}$ be the centers of the boxes in $S_{+}$that contain $p$. Assume w.l.o.g. that

$$
\delta:=d_{\infty}\left(p, b_{1}\right)=\max \left\{d_{\infty}\left(p, b_{i}\right): 1 \leq i \leq m\right\} .
$$

Let $B$ be the box of $S_{+}$having $b_{1}$ as its center. We claim that $d_{\infty}\left(b_{1}, b_{i}\right)<\delta$ for $1 \leq i \leq m$. Indeed, let $1 \leq j \leq D$. The $j$-th coordinate $b_{i j}$ of $b_{i}$ satisfies $0<b_{i j} \leq \delta$. As a result, $\left|b_{1 j}-b_{i j}\right|<\delta$. This proves the claim.

Since $B$ contains $p$, it has sides of length at least $2 \delta$. Therefore, since each center $b_{i}$ has $L_{\infty}$-distance less than $\delta$ to $b_{1}$, these centers are contained in the interior of $B$. Then, the constant overlap property implies that $m \leq c_{D}$.

This proves that $p$ is contained in at most $c_{D}$ boxes of $S_{+}$. By a symmetric argument, $p$ is contained in at most $c_{D}$ boxes of $S$ that have their centers in a fixed 
$D$-dimensional quadrant. Hence, $p$ is contained in at most $2^{D} c_{D}$ boxes whose centers have non-zero coordinates.

Let $S_{0}$ be the set of all boxes in $S$ whose centers have zero as their first coordinate. Consider the set $S_{0}^{\prime}$ of $(D-1)$-dimensional boxes obtained from $S_{0}$ by deleting from each box its first coordinates. The constant overlap property for $S_{0}$ implies that the boxes of $S_{0}^{\prime}$ are also of constant overlap, with constant $c_{D}$. Hence, by the induction hypothesis, point $p^{\prime}=\left(p_{2}, \ldots, p_{D}\right)$ is contained in at most $2^{1+(D-1)^{2}} c_{D}$ boxes of $S_{0}^{\prime}$. These boxes of $S_{0}^{\prime}$ correspond exactly to the boxes of $S_{0}$ that contain $p$.

Hence, at most $2^{1+(D-1)^{2}} c_{D}$ boxes of $S_{0}$ contain $p$. It follows from a symmetric argument that for each $1 \leq i \leq D$, point $p$ is contained in at most $2^{1+(D-1)^{2}} c_{D}$ boxes whose centers have zero as their $i$-th coordinate.

To summarize, we have shown that there are at most

$$
2^{D} c_{D}+D 2^{1+(D-1)^{2}} c_{D} \leq 2^{1+D^{2}} c_{D}
$$

boxes of $S$ that contain $p$. This completes the proof.

In the next two subsections, we shall give two solutions for the box containment problem.

\subsection{A solution based on segment trees}

We start with the one-dimensional case. Let $S$ be a set of $n$ intervals $\left[a_{j}: b_{j}\right], 1 \leq j \leq$ $n$, that are of constant overlap with constant $c$.

The one-dimensional structure: We store the intervals in the leaves of a balanced binary search tree $T$, sorted by their right endpoints. The leaves of this tree are threaded in a doubly-linked list.

The query algorithm: Let $p \in \mathbb{R}$ be a query element. Search in $T$ for the leftmost leaf containing a right endpoint that is at least equal to $p$. Starting in this leaf, walk along the leaves to the right and report all intervals encountered that contain $p$. Stop walking as soon as $4 c$ intervals have been reported, or $c$ intervals have been encountered that do not contain $p$.

Lemma 9 The above data structure solves the one-dimensional containment problem in a set of $n$ intervals of constant overlap in $O(n)$ space with a query time of $O(\log n)$. Intervals can be inserted and deleted in $O(\log n)$ time.

Proof: The complexity bounds are clear; we only have to prove that the query algorithm is correct. Clearly, all intervals that are reported contain $p$. It remains to show that all intervals containing $p$ are reported.

Let $v$ be the leaf of $T$ in which the search for $p$ ends. The algorithm starts in $v$ and walks to the right. First note that all leaves to the left of $v$ store intervals that do not contain $p$. We know from Lemma 8 that there are at most $4 c$ intervals that contain $p$. Hence, after $4 c$ intervals have been reported, the algorithm can stop. Now assume that in leaf $w$, we encounter the $c$-th interval that does not contain $p$. All $c$ 
encountered intervals that do not contain $p$ lie completely to the right of $p$. If there is a leaf to the right of $w$ whose interval contains $p$, then this interval contains these $c$ intervals. By the constant overlap property, such a leaf cannot exist. This proves that the algorithm can stop in leaf $w$, i.e., if it has encountered $c$ intervals that do not contain $p$.

We next consider the $D$-dimensional case, where $D \geq 2$. Let $S$ be a set of $n$ boxes in $\mathbb{R}^{D}$ that are of constant overlap with constant $c_{D}$. The data structure is a segment tree for the intervals of the first coordinates of the boxes. (See $[13,15]$.) The nodes of this tree contain appropriate associated structures.

If $B=B_{1} \times B_{2} \times \ldots \times B_{D}$ is a box in $\mathbb{R}^{D}$, then $B^{\prime}$ denotes the box $B_{2} \times \ldots \times B_{D}$ in $\mathbb{R}^{D-1}$. Similarly, $S^{\prime}$ denotes the set $\left\{B^{\prime}: B \in S\right\}$. Recall that we use a similar notation for points.

The $D$-dimensional structure for $D \geq 2$ : Let $a_{1}<a_{2}<\ldots<a_{m}$, where $m \leq 2 n$, be the sorted sequence of all distinct endpoints of the intervals of the first coordinates of the boxes in $S$. We store the elementary intervals

$$
\left(-\infty: a_{1}\right),\left[a_{1}: a_{1}\right],\left(a_{1}: a_{2}\right),\left[a_{2}: a_{2}\right], \ldots,\left(a_{m-1}: a_{m}\right),\left[a_{m}: a_{m}\right],\left(a_{m}: \infty\right)
$$

in this order in the leaves of a balanced binary search tree, called the main tree. Each node $v$ of this tree has associated with it an interval $I_{v}$ being the union of the elementary intervals of the leaves in the subtree of $v$. Let $S_{v}$ be the set of all boxes $B=B_{1} \times B_{2} \times \ldots \times B_{D}$ in $S$ such that $B_{1}$ spans the interval associated with $v$ but does not span the interval associated with its father node, i.e. $I_{v} \subseteq B_{1}$ and $I_{f(v)} \not B_{1}$, where $f(v)$ is the father of $v$.

Each node $v$ of the main tree contains (a pointer to) an associated structure for the set $S_{v}$ : Partition this set into $S_{v l}, S_{v c}$ and $S_{v r}$, consisting of those boxes of $S_{v}$ whose centers lie to the left of the "vertical" slab $I_{v} \times \mathbb{R}^{D-1}$, in or on the boundary of this slab, and to the right of this slab, respectively.

The associated structure of $v$ consists of three recursively defined $(D-1)$-dimensional structures for the sets $S_{v l}^{\prime}, S_{v c}^{\prime}$ and $S_{v r}^{\prime}$.

Remark: The interval $I_{v}$ may be open, half-open or closed. Therefore, the boundary of the slab $I_{v} \times \mathbb{R}^{D-1}$ does not necessarily belong to this slab.

The query algorithm: Let $p=\left(p_{1}, p_{2}, \ldots, p_{D}\right) \in \mathbb{R}^{D}$ be a query point. Search in the main tree for the elementary interval that contains $p_{1}$. For each node $v$ on the search path, recursively perform a $(D-1)$-dimensional query with the point $p^{\prime}$ in the three structures that are stored with $v$.

At the last level of the recursion, a one-dimensional query is performed in a binary search tree. In this tree, the algorithm stops if it has reported $2^{1+D^{2}} c_{D}$ boxes of $S$ that contain $p$, or if it has encountered $c_{D}$ boxes of $S$ that do not contain $p$. (If in this tree $c_{D}$ boxes that do not contain $p$ have been encountered, then the algorithm only stops at this level of the recursion. If, on the other hand, overall $2^{1+D^{2}} c_{D}$ boxes that contain $p$ have been reported, then the entire query algorithm stops.)

The correctness proof of this algorithm uses the following lemma. 
Lemma 10 Let $x$ and $y$ be real numbers, let $p \in \mathbb{R}^{D}$ and let $i, 1 \leq i \leq D-1$, be an integer such that $x \leq p_{i} \leq y$. Let $A=\left[a_{1}: a_{1}+\alpha\right] \times \ldots \times\left[a_{D}: a_{D}+\alpha\right]$ and $B=\left[b_{1}: b_{1}+\beta\right] \times \ldots \times\left[b_{D}: b_{D}+\beta\right]$ be two boxes such that $a_{i} \leq x, a_{i}+\alpha \geq y, b_{i} \leq x$, $b_{i}+\beta \geq y, b_{D}>p_{D}$ and $b_{D}+\beta \leq a_{D}+\alpha$. Finally, assume that $p$ is contained in $A$. Then,

1. $a_{D}<b_{D}+\beta / 2<a_{D}+\alpha$,

2. if one of the following three conditions holds,

(a) $x \leq a_{i}+\alpha / 2 \leq y$ and $x \leq b_{i}+\beta / 2 \leq y$,

(b) $a_{i}+\alpha / 2<x$ and $b_{i}+\beta / 2<x$,

(c) $a_{i}+\alpha / 2>y$ and $b_{i}+\beta / 2>y$,

then $a_{i}<b_{i}+\beta / 2<a_{i}+\alpha$.

Proof: The right inequality of the first assertion follows easily: $b_{D}+\beta / 2<b_{D}+\beta \leq$ $a_{D}+\alpha$. Since $p$ is contained in $A$, we have $a_{D} \leq p_{D}$. Therefore, $a_{D} \leq p_{D}<b_{D}<$ $b_{D}+\beta / 2$. This proves 1 .

Before we prove 2 , observe that $b_{D}+\beta \leq a_{D}+\alpha<b_{D}+\alpha$. It follows that $\beta<\alpha$.

Assume that case 2(a) applies, i.e., $x \leq a_{i}+\alpha / 2 \leq y$ and $x \leq b_{i}+\beta / 2 \leq y$. Since $b_{i}+\beta / 2 \leq y \leq a_{i}+\alpha$ and $b_{i}+\beta / 2 \geq x \geq a_{i}$, we only have to show that $a_{i} \neq b_{i}+\beta / 2$ and $b_{i}+\beta / 2 \neq a_{i}+\alpha$.

Assume that $a_{i}=b_{i}+\beta / 2$. Then, $x=a_{i}=b_{i}+\beta / 2$. First note that $\alpha / 2 \leq$ $y-a_{i}=y-x$. Since $\beta / 2=b_{i}+\beta-\left(b_{i}+\beta / 2\right) \geq y-\left(b_{i}+\beta / 2\right)=y-x$, we infer that $\alpha \leq \beta$. This is a contradiction and, hence, $a_{i}<b_{i}+\beta / 2$.

Next assume that $b_{i}+\beta / 2=a_{i}+\alpha$. Then, $y=b_{i}+\beta / 2=a_{i}+\alpha$ and in a similar way we can prove that $\alpha \leq \beta$. Therefore, $b_{i}+\beta / 2<a_{i}+\alpha$.

Next consider case 2(b), i.e., assume that $a_{i}+\alpha / 2<x$ and $b_{i}+\beta / 2<x$. Since $b_{i}+\beta / 2<x \leq y \leq a_{i}+\alpha$, we only have to show that $a_{i}<b_{i}+\beta / 2$. We prove this by contradiction. So, assume that $a_{i} \geq b_{i}+\beta / 2$. Then, $\alpha / 2=a_{i}+\alpha / 2-a_{i}<x-a_{i} \leq$ $x-\left(b_{i}+\beta / 2\right)=x-b_{i}-\beta / 2$. Since $b_{i}+\beta \geq y \geq x$, we get $x-b_{i} \leq \beta$. Therefore, $\alpha / 2<x-b_{i}-\beta / 2 \leq \beta-\beta / 2=\beta / 2$, i.e., $\alpha<\beta$. This is a contradiction and, hence, we have proved that $a_{i}<b_{i}+\beta / 2$.

Case 2(c) can be treated in the same way as case $2(\mathrm{~b})$.

Lemma 11 The query algorithm is correct.

Proof: It is well known that the set of all boxes $B=B_{1} \times \ldots \times B_{D}$ such that $p_{1} \in B_{1}$ is exactly the union of all sets $S_{v}$, where $v$ is a node on the search path to $p_{1}$. Hence, we only have to consider the nodes on this search path.

Let $v$ be a node on the path to $p_{1}$. We know that $p_{1}$ is contained in the first interval of each box of $S_{v}$. Hence, we have to find all boxes in $S_{v}$ whose last $D-1$ intervals contain $\left(p_{2}, \ldots, p_{D}\right)$. We claim that the recursive queries in the three structures stored with $v$ find these boxes. By Lemma 8 , there are at most $2^{1+D^{2}} c_{D}$ boxes that contain $p$. Hence, at the last level of the recursion, the query algorithm can stop as soon as it has reported this many boxes. It remains to prove that, at the last level, the query algorithm can stop if it has encountered $c_{D}$ boxes that do not contain $p$.

Consider such a last level. That is, let $v_{1}, v_{2}, \ldots, v_{D-1}$ be nodes such that 
1. $v_{1}=v$,

2. $v_{i}$ is a node of the main tree of one of the three structures that are stored with $v_{i-1}$,

3. $v_{i}$ lies on the search path to $p_{i}$.

The algorithm makes one-dimensional queries with $p_{D}$ in the three structures-binary search trees - that are stored with $v_{D-1}$.

Consider one such query. The algorithm searches for $p_{D}$. Let $r$ be the leaf in which this search ends. Starting in $r$, the algorithm walks along the leaves to the right. During this walk, it encounters boxes that do or do not contain $p$. Assume that in leaf $s$, the $c_{D}$-th box is encountered that does not contain $p$. We have to show that the algorithm can stop in $s$. That is, we must show that all leaves to the right of $s$ store boxes that do not contain $p$.

Assume this is not the case. Then, there is a box

$$
A=\left[a_{1}: a_{1}+\alpha\right] \times\left[a_{2}: a_{2}+\alpha\right] \times \ldots \times\left[a_{D}: a_{D}+\alpha\right]
$$

that is stored in a leaf to the right of $s$ and that contains $p$. Let

$$
B^{(j)}=\left[b_{j 1}: b_{j 1}+\beta_{j}\right] \times\left[b_{j 2}: b_{j 2}+\beta_{j}\right] \times \ldots \times\left[b_{j D}: b_{j D}+\beta_{j}\right], 1 \leq j \leq c_{D},
$$

be the encountered boxes between $r$ and $s$ that do not contain $p$. We shall prove that $A$ contains the centers of all these boxes in its interior. This will be a contradiction.

Let $1 \leq j \leq c_{D}$. Since the leaf of $A$ lies to the right of the leaf of $B^{(j)}$, we know that $b_{j D}+\beta_{j} \leq a_{D}+\alpha$.

Let $1 \leq i \leq D-1$ and let $x \leq y$ be the boundary points of the interval $I_{v_{i}}$. Then, $x \leq p_{i} \leq y$, because $p_{i} \in I_{v_{i}}$. Moreover, we know that $I_{v_{i}}$ is contained in the $i$-th interval of $A$ and $B^{(j)}$, i.e., $a_{i} \leq x, a_{i}+\alpha \geq y, b_{j i} \leq x$ and $b_{j i}+\beta_{j} \geq y$. Finally, since the leaf of $B^{(j)}$ is equal to leaf $r$ or lies to the right of it, we know that $p_{D} \leq b_{j D}+\beta_{j}$. Since $B^{(j)}$ does not contain $p$, this implies that $b_{j D}>p_{D}$. (Note that the $i$-th interval of $B^{(j)}$ contains $p_{i}, 1 \leq i \leq D-1$.)

The definition of our data structure implies that the $i$-th coordinates of the centers of $A$ and $B^{(j)}$ are either both on the boundary or contained in $I_{v_{i}}$, or both are less than $x$, or both are larger than $y$. Therefore, all requirements of Lemma 10 are satisfied and we conclude that $a_{i}<b_{j i}+\beta_{j} / 2<a_{i}+\alpha$.

Since $i$ was arbitrary between one and $D-1$, and since Lemma 10 also implies that $a_{D}<b_{j D}+\beta_{j} / 2<a_{D}+\alpha$, we have proved that the center of $B^{(j)}$ is contained in the interior of $A$. This completes the proof.

We analyze the complexity of the $D$-dimensional structure. Let $M(n, D)$ denote the size of the data structure. It is well known that the first interval of a box in $S$ is stored in the associated structure of $O(\log n)$ nodes of the main tree. This implies that $M(n, D)=O(M(n, D-1) \log n)$. Since $M(n, 1)=O(n)$, we get $M(n, D)=$ $O\left(n(\log n)^{D-1}\right)$. Using presorting, the structure can be built in $O\left(n(\log n)^{D-1}\right)$ time.

Let $Q(n, D)$ denote the query time. Then $Q(n, 1)=O(\log n)$. The query algorithm performs $(D-1)$-dimensional queries in each of the $O(\log n)$ nodes on the search path. 
As a result, the query time satisfies $Q(n, D)=O(Q(n, D-1) \log n)$, which solves to $O\left((\log n)^{D}\right)$. By applying fractional cascading (see [6]) in the 2-dimensional case, we decrease $Q(n, 2)$ to $O(\log n)$. This improves the query time to $Q(n, D)=O\left((\log n)^{D-1}\right)$ for $D \geq 2$.

By applying standard techniques, the data structure can be adapted to handle insertions and deletions of boxes. (See $[13,14]$.) Because of dynamic fractional cascading, the query time increases by a factor of $O(\log \log n)$, whereas the size only increases by a constant factor. The amortized update time is $O\left((\log n)^{D-1} \log \log n\right)$.

We summarize our result.

Theorem 4 Let $S$ be a set of $n$ boxes in $\mathbb{R}^{D}$ of constant overlap. There exists a data structure of size $O\left(n(\log n)^{D-1}\right)$ such that for any point $p \in \mathbb{R}^{D}$, we can find all boxes of $S$ that contain $p$ in $O\left((\log n)^{D-1}\right)$ time. This static data structure can be built in $O\left(n(\log n)^{D-1}\right)$ time.

For the dynamic version of the problem, the query time is $O\left((\log n)^{D-1} \log \log n\right)$ and the size of the structure is $O\left(n(\log n)^{D-1}\right)$. Boxes can be inserted and deleted in $O\left((\log n)^{D-1} \log \log n\right)$ amortized time.

\subsection{A solution based on skewer trees}

In this section, we give a linear space solution to the box containment problem. This solution is based on skewer trees, introduced by Edelsbrunner et al. [8]. (See also Smid [21] for a dynamic version of this data structure.)

Let $S$ be a set of $n$ boxes in $\mathbb{R}^{D}$ that are of constant overlap with constant $c_{D}$. For $D=1$, the data structure is the same as in the previous subsection.

The $D$-dimensional structure for $D \geq 2$ : If $S$ is empty, then the data structure is also empty. Assume that $S$ is non-empty. Let $\gamma$ be the median of the set

$$
\left\{\left(a_{1}+b_{1}\right) / 2:\left[a_{1}: b_{1}\right] \times\left[a_{2}: b_{2}\right] \times \ldots \times\left[a_{D}: b_{D}\right] \in S\right\}
$$

and let $\epsilon$ be the largest element that is less than $\gamma$ in the set of all elements $a_{1}$, $\left(a_{1}+b_{1}\right) / 2$ and $b_{1}$, where $\left[a_{1}: b_{1}\right] \times \ldots \times\left[a_{D}: b_{D}\right]$ ranges over $S$. Let $\gamma_{1}:=\gamma-\epsilon / 2$ and let $\sigma$ be the hyperplane in $\mathbb{R}^{D}$ with equation $x_{1}=\gamma_{1}$.

Let $S_{<}, S_{0}$ and $S_{>}$be the set of boxes $\left[a_{1}: b_{1}\right] \times \ldots \times\left[a_{D}: b_{D}\right]$ in $S$ such that $b_{1}<\gamma_{1}, a_{1} \leq \gamma_{1} \leq b_{1}$ and $\gamma_{1}<a_{1}$, respectively. The $D$-dimensional data structure for the set $S$ is an augmented binary search tree - called the main tree-having the following form:

1. The root contains the hyperplane $\sigma$.

2. The root contains pointers to its left and right sons, which are $D$-dimensional structures for the sets $S_{<}$and $S_{>}$, respectively.

3. The root contains (a pointer to) an associated structure for the set $S_{0}$ : Partition this set into $S_{0 l}$ and $S_{0 r}$, consisting of those boxes in $S_{0}$ whose centers lie to the left of the hyperplane $\sigma$, and to the right of $\sigma$, respectively.

The associated structure of the root consists of two $(D-1)$-dimensional structures for the sets $S_{0 l}^{\prime}$ and $S_{0 r}^{\prime}$. 
Remark: By our choice of $\gamma_{1}$, the hyperplane $\sigma$ does not contain the center of any box in $S$. Moreover, each of the sets $S_{<}$and $S_{>}$has size at most $n / 2$. The set $S_{0}$ may have size $n$. The height of the data structure is defined as the height of its main tree. It follows that the structure has height $O(\log n)$.

The query algorithm: Let $p=\left(p_{1}, p_{2}, \ldots, p_{D}\right) \in \mathbb{R}^{D}$ be a query point. Let $\sigma$ : $x_{1}=\gamma_{1}$ be the hyperplane stored in the root of the main tree. Recursively perform a $(D-1)$-dimensional query with the point $p^{\prime}$ in the two structures that are stored with the root.

If $p_{1}<\gamma_{1}$ (resp. $p_{1}>\gamma_{1}$ ), then recursively perform a $D$-dimensional query with $p$ in the left (resp. right) subtree of the root, unless this subtree is empty, in which case the algorithm stops.

At the last level of the recursion, a one-dimensional query is performed in a binary search tree. In this tree, the algorithm stops if it has reported $2^{1+D^{2}} c_{D}$ boxes of $S$ that contain $p$, or if it has encountered $c_{D}$ boxes of $S$ whose last intervals do not contain $p_{D}$, or if it has encountered $2^{1+D^{2}} c_{D}$ boxes of $S$ that do not contain $p$ but whose last intervals contain $p_{D}$.

The correctness proof is similar to the one of Subsection 5.1. Again, we start with a technical lemma.

Lemma 12 Let $p \in \mathbb{R}^{D}$ and let $\sigma_{i}: x_{i}=\gamma_{i}, 1 \leq i \leq D-1$, be hyperplanes in $\mathbb{R}^{D}$. Let $A=\left[a_{1}: a_{1}+\alpha\right] \times \ldots \times\left[a_{D}: a_{D}+\alpha\right]$ and $B=\left[b_{1}: b_{1}+\beta\right] \times \ldots \times\left[b_{D}: b_{D}+\beta\right]$ be two boxes such that $b_{D}>p_{D}$ and $b_{D}+\beta \leq a_{D}+\alpha$. Assume that $p$ is contained in A. Finally, assume that $a_{i}+\alpha / 2<\gamma_{i} \leq a_{i}+\alpha$ and $b_{i}+\beta / 2<\gamma_{i} \leq b_{i}+\beta$ for all $1 \leq i \leq D-1$.

Then $a_{i}<b_{i}+\beta / 2<a_{i}+\alpha$ for all $1 \leq i \leq D$.

Proof: We start with $i=D$. It follows directly that $b_{D}+\beta / 2<b_{D}+\beta \leq a_{D}+\alpha$. Since $p$ is contained in $A$, we have $a_{D} \leq p_{D}$. Therefore, $a_{D} \leq p_{D}<b_{D}<b_{D}+\beta / 2$.

Let $1 \leq i \leq D-1$. Then $b_{i}+\beta / 2<\gamma_{i} \leq a_{i}+\alpha$. It remains to show that $a_{i}<b_{i}+\beta / 2$. Assume that $b_{i}+\beta / 2 \leq a_{i}$. Then, $\beta / 2=b_{i}+\beta-\left(b_{i}+\beta / 2\right) \geq$ $\gamma_{i}-\left(b_{i}+\beta / 2\right) \geq \gamma_{i}-a_{i}>\alpha / 2$, i.e., $\beta>\alpha$. But, since $b_{D}+\beta \leq a_{D}+\alpha<b_{D}+\alpha$, we also have $\beta<\alpha$. This is a contradiction.

\section{Lemma 13 The query algorithm is correct.}

Proof: It is clear that the algorithm branches correctly. Hence, we only have to consider the last level of the recursion. Since there are at most $2^{1+D^{2}} c_{D}$ boxes that contain $p$, the algorithm can stop as soon as it has reported this many boxes. It remains to prove that, at the last level, the query algorithm can stop if it has encountered $c_{D}$ boxes whose last intervals do not contain $p_{D}$, or if it has encountered $2^{1+D^{2}} c_{D}$ boxes that do not contain $p$ but whose last intervals contain $p_{D}$.

Consider such a last level. That is, let $v_{1}, v_{2}, \ldots, v_{D-1}$ be nodes such that

1. $v_{1}$ is a node of the main tree, 
2. $v_{i}$ is a node of the main tree of one of the two structures that are stored with $v_{i-1}$,

3. $v_{i}$ lies on the search path to $p_{i}$.

Let $\sigma_{i}: x_{i}=\gamma_{i}$ be the hyperplane that is stored with $v_{i}, 1 \leq i \leq D-1$.

The algorithm makes one-dimensional queries with $p_{D}$ in the two structuresbinary trees - that are stored with $v_{D-1}$. Consider one such query. The algorithm searches for $p_{D}$. Let $r$ be the leaf in which this search ends. Starting in $r$, the algorithm walks along the leaves to the right. Assume that in leaf $s$, the $c_{D}$-th box is encountered whose last interval does not contain $p_{D}$, or the $\left(2^{1+D^{2}} c_{D}\right)$-th box is encountered that does not contain $p$ but whose last interval contains $p_{D}$. We will prove that all leaves to the right of $s$ store boxes that do not contain $p$.

Assume this is not the case. Then, there is a box

$$
A=\left[a_{1}: a_{1}+\alpha\right] \times\left[a_{2}: a_{2}+\alpha\right] \times \ldots \times\left[a_{D}: a_{D}+\alpha\right]
$$

that is stored in a leaf to the right of $s$ and that contains $p$. Let

$$
B^{(j)}=\left[b_{j 1}: b_{j 1}+\beta_{j}\right] \times\left[b_{j 2}: b_{j 2}+\beta_{j}\right] \times \ldots \times\left[b_{j D}: b_{j D}+\beta_{j}\right]
$$

be the encountered boxes between $r$ and $s$ that do not contain $p$.

The definition of our data structure implies that for each $1 \leq i \leq D-1$, the centers of $A$ and the $B^{(j)}$ 's lie on the same side of the hyperplane $\sigma_{i}$. Moreover, these boxes intersect $\sigma_{i}$. We assume w.l.o.g. that for all $1 \leq i \leq D-1, a_{i}+\alpha / 2<\gamma_{i} \leq a_{i}+\alpha$, and for all $j$ and all $1 \leq i \leq D-1, b_{j i}+\beta_{j} / 2<\gamma_{i} \leq b_{j i}+\beta_{j}$.

There are two possible cases. First assume that the last intervals of $c_{D}$ boxes $B^{(j)}$ do not contain $p_{D}$. Consider such a box $B^{(j)}$. Since the leaf of this box is equal to leaf $r$ or lies to the right of it, we must have $p_{D} \leq b_{j D}+\beta_{j}$. Hence, $p_{D}<b_{j D}$. Also, since the leaf of $A$ lies to the right of the leaf of $B^{(j)}$, we have $b_{j D}+\beta_{j} \leq a_{D}+\alpha$. Hence, all requirements of Lemma 12 are satisfied. We conclude that the center of $B^{(j)}$ is contained in the interior of $A$. This proves that the interior of $A$ contains more than $c_{D}$ centers, namely its own center and the centers of $c_{D}$ boxes $B^{(j)}$. This is a contradiction.

The second case is where $2^{1+D^{2}} c_{D}$ boxes $B^{(j)}$ do not contain $p$ but their last intervals contain $p_{D}$. We claim that the point $q:=\left(\gamma_{1}, \gamma_{2}, \ldots, \gamma_{D-1}, p_{D}\right)$ is contained in $A$ and in all these $B^{(j)}$ 's. To prove this, first note that $a_{D} \leq p_{D} \leq a_{D}+\alpha$, because $A$ contains $p$. Consider any of these boxes $B^{(j)}$. By our assumption, $b_{j D} \leq p_{D} \leq b_{j D}+\beta_{j}$. Our assumptions also imply that $a_{i} \leq \gamma_{i} \leq a_{i}+\alpha$ and $b_{j i} \leq \gamma_{i} \leq b_{j i}+\beta_{j}$ for all $1 \leq i \leq D-1$. Hence, there are more than $2^{1+D^{2}} c_{D}$ boxes that contain $q$. This contradicts Lemma 8.

The complexity analysis is similar to that of the previous subsection. Since the main tree has height $O(\log n)$, the query time is bounded by $O\left((\log n)^{D}\right)$. In the planar case, we can apply fractional cascading to improve the query time to $O(\log n)$. Then, the query time for the $D$-dimensional case is improved to $O\left((\log n)^{D-1}\right)$. It is easy to see that the data structure has size $O(n)$ and that it can be built in $O(n \log n)$ time. (See [8] for details.) 
We can adapt the data structure such that it can also handle insertions and deletions of boxes. Since the algorithms and their running times are exactly the same as in [21], we refer the reader to that paper for the details. Because of dynamic fractional cascading, the query time increases by a factor of $O(\log \log n)$, whereas the size only increases by a constant factor. The amortized update time is $O\left((\log n)^{2} \log \log n\right)$.

We summarize our result.

Theorem 5 Let $S$ be a set of $n$ boxes in $\mathbb{R}^{D}$ of constant overlap. There exists a data structure of size $O(n)$ such that for any point $p \in \mathbb{R}^{D}$, we can find all boxes of $S$ that contain $p$ in $O\left((\log n)^{D-1}\right)$ time. This static data structure can be built in $O(n \log n)$ time.

For the dynamic version of the problem, the query time is $O\left((\log n)^{D-1} \log \log n\right)$ and the size of the structure is $O(n)$. Boxes can be inserted and deleted in amortized time $O\left((\log n)^{2} \log \log n\right)$.

\section{Maintaining the closest pair}

In this section, we apply the results obtained so far to maintain a closest pair of a point set under insertions and deletions. Let $S$ be a set of $n$ points in $\mathbb{R}^{D}$ and let $1 \leq t \leq \infty$ be a real number. We denote the $L_{t}$-distance between any two points $p$ and $q$ in $\mathbb{R}^{D}$ by $d(p, q)$. The pair $P, Q \in S$ is called a closest pair of $S$ if

$$
d(P, Q)=\min \{d(p, q): p, q \in S, p \neq q\} .
$$

We introduce the following notations. For any point $p \in \mathbb{R}^{D}$, box $(p)$ denotes the smallest box centered at $p$ that contains at least $(2 D+2)^{D}$ points of $S \backslash\{p\}$. In other words, the side length of $\operatorname{box}(p)$ is twice the $L_{\infty}$-distance between $p$ and its $\left(1+(2 D+2)^{D}\right)$-th $\left(\right.$ resp. $(2 D+2)^{D}$-th) $L_{\infty}$-neighbor, if $p \in S$ (resp. $\left.p \notin S\right)$.

Let $N(p)$ be the set of points of $S \backslash\{p\}$ that are contained in the interior of box $(p)$. Note that $N(p)$ has size less than $(2 D+2)^{D}$. In fact, $N(p)$ may even be empty.

Our data structure is based on the following lemma.

Lemma 14 The set $\{(p, q): p \in S, q \in N(p)\}$ contains a closest pair of $S$.

Proof: Let $(P, Q)$ be a closest pair of $S$. We have to show that $Q \in N(P)$. Assume this is not the case. Let $\delta$ be the side length of box $(P)$. Since $Q$ lies outside or on the boundary of this box, we have $d(P, Q) \geq \delta / 2$.

Partition box $(P)$ into $(2 D+2)^{D}$ subboxes with sides of length $\delta /(2 D+2)$. Since box $(P)$ contains at least $1+(2 D+2)^{D}$ points of $S$, one of these subboxes contains at least two points. These two points have distance at most $D \cdot \delta /(2 D+2)<\delta / 2$, which is a contradiction, because $(P, Q)$ is a closest pair of $S$.

The set $\{b o x(p): p \in S\}$ is of constant overlap: each box contains the centers of at most $(2 D+2)^{D}$ boxes in its interior. These centers are precisely the points of $N(p) \cup\{p\}$. This fact and the above lemma suggest the following data structure. 


\section{The closest pair data structure:}

1. The points of $S$ are stored in a range tree.

2. The distances of the multiset $\{d(p, q): p \in S, q \in N(p)\}$ are stored in a heap. With each distance, we store the corresponding pair of points. (Note that both $d(p, q)$ and $d(q, p)$ may occur in the heap.)

3. The points of $S$ are stored in a dictionary. With each point $p$, we store a list containing the elements of $N(p)$. For convenience, we also call this list $N(p)$. With each point $q$ in $N(p)$, we store a pointer to the occurrence of $d(p, q)$ in the heap.

4. The set $\{b o x(p): p \in S\}$ is stored in the dynamic data structure of Theorem 4 . This structure is called the box tree.

It follows from Lemma 14 that the pair of points that is stored with the minimal element of the heap is a closest pair of $S$. The update algorithms are rather straightforward.

The insertion algorithm: Let $p \in \mathbb{R}^{D}$ be the point to be inserted. Assume w.l.o.g. that $p \notin S$.

1. Using the range tree, find the $(2 D+2)^{D} L_{\infty}$-neighbors of $p$ in $S$. The point among these neighbors having maximal $L_{\infty}$-distance to $p$ determines box $(p)$. The neighbors that are contained in the interior of box $(p)$ form the list $N(p)$.

2. Insert $p$ into the range tree and insert the distances $d(p, q), q \in N(p)$ into the heap. Then, insert $p$-together with the list $N(p)$-into the dictionary. With each point $q$ in $N(p)$, store a pointer to $d(p, q)$ in the heap. Finally, insert box $(p)$ into the box tree.

3. Using the box tree, find all boxes that contain $p$. For each reported element $b o x(q), q \neq p$, that contains $p$ in its interior, do the following:

(a) Search in the dictionary for $q$. Insert $p$ into $N(q)$, insert $d(q, p)$ into the heap and store with $p$ a pointer to $d(q, p)$.

(b) If $N(q)$ has size less than $(2 D+2)^{D}$, then the insertion algorithm is completed. Otherwise, if $N(q)$ has size $(2 D+2)^{D}$, let $r_{1}, \ldots, r_{l}$ be all points in $N(q)$ that have maximal $L_{\infty}$-distance to $q$. For each $1 \leq i \leq l$, delete $r_{i}$ from $N(q)$ and delete $d\left(q, r_{i}\right)$ from the heap. Finally, delete box $(q)$ from the box tree and insert the box centered at $q$ having $r_{1}$ on its boundary as the new box $(q)$.

The deletion algorithm: Let $p \in S$ be the point to be deleted.

1. Delete $p$ from the range tree. Search for $p$ in the dictionary. For each point $q$ in $N(p)$, delete the distance $d(p, q)$ from the heap. Then, delete $p$ and $N(p)$ from the dictionary. Finally, delete box $(p)$ from the box tree. 
2. Using the box tree, find all boxes that contain $p$. For each reported element box $(q)$, do the following:

(a) If $p$ lies in the interior of box $(q)$, then search in the dictionary for $q$, delete $p$ from $N(q)$ and delete $d(q, p)$ from the heap.

(b) Using the range tree, find the $1+(2 D+2)^{D} L_{\infty}$-neighbors of $q$. Let box $(q)$ be the smallest box centered at $q$ that contains these neighbors. If $b o x(q)=$ $\operatorname{box}_{0}(q)$, then the deletion algorithm is completed.

(c) Otherwise, if $\operatorname{box}(q) \neq b_{b o x}(q)$, let $r_{1}, \ldots, r_{l}$ be all points that are contained in the interior of $b o x_{0}(q)$ but that do not belong to $N(q) \cup\{q\}$. For each $1 \leq i \leq l$, insert $r_{i}$ into $N(q)$, insert $d\left(q, r_{i}\right)$ into the heap and store with $r_{i}$ a pointer to $d\left(q, r_{i}\right)$. Finally, delete $\operatorname{box}(q)$ from the box tree and insert $\operatorname{box}_{0}(q)$, being the new box $(q)$.

It is easy to verify that these update algorithms correctly maintain the closest pair data structure. During these algorithms, we perform a constant number of query and update operations in the range tree, the box tree, the heap and the dictionary. Therefore, by Theorems 2 and 4, the amortized update time of the entire data structure is bounded by $O\left((\log n)^{D-1} \log \log n\right)$. We have proved the following result.

Theorem 6 Let $S$ be a set of $n$ points in $\mathbb{R}^{D}$ and let $1 \leq t \leq \infty$. There exists a data structure of size $O\left(n(\log n)^{D-1}\right)$ that maintains an $L_{t}$-closest pair of $S$ in $O\left((\log n)^{D-1} \log \log n\right)$ amortized time per insertion and deletion. The constant factor in the space (resp. update time) bound is of the form $O(D)^{D}\left(\right.$ resp. $\left.2^{O\left(D^{2}\right)}\right)$.

Consider again our data structure. The box $\operatorname{box}(p)$ that is associated with point $p$ contains an $L_{\infty}$-neighbor of $p$ in $S \backslash\{p\}$. Therefore, the data structure can easily be adapted such that it maintains for each point in $S$ its $L_{\infty}$-neighbor.

Corollary 1 Let $S$ be a set of $n$ points in $\mathbb{R}^{D}$. There exists a data structure of size $O\left(n(\log n)^{D-1}\right)$ that maintains an $L_{\infty}$-neighbor of each point in $S$. This data structure has an amortized update time of $O\left((\log n)^{D-1} \log \log n\right)$.

\section{A transformation for reducing the space com- plexity}

The closest pair data structure of the previous section uses more than linear space. This raises the question if the same update time can be obtained using only linear space. In this section, we show that for $D \geq 3$, this is indeed possible. For $D=2$, we will obtain a family of closest pair data structures.

Note that we have a linear space solution for maintaining the set $\{b o x(p): p \in S\}$. (See Theorem 5.) For the $L_{\infty}$-neighbor problem, however, no linear space solution having polylogarithmic query and update times is known. Hence, in order to reduce the space complexity, we should avoid using the range tree. 
We will give a transformation that, given any dynamic closest pair data structure having more than linear size, produces another dynamic closest pair structure that uses less space.

The transformed data structure is composed on two sets $A$ and $B$ that partition $S$. The set $B$ is contained in a dynamic data structure. To reduce space, $B$ is a subset of the entire set and contains points involved in $o(n)$ updates only.

Let $D S$ be a data structure that maintains a closest pair in a set of $n$ points in $\mathbb{R}^{D}$ under insertions and deletions. Let $S(n)$ and $U(n)$ denote the size and update time of $D S$, respectively. The update time may be worst-case or amortized. We assume that $S(n)$ and $U(n)$ are non-decreasing and smooth in the sense that $S(\Theta(n))=$ $\Theta(S(n))$ and $U(\Theta(n))=\Theta(U(n))$. Finally, let $f(n)$ be a non-decreasing smooth integer function. such that $1 \leq f(n) \leq n / 2$.

Let $S \subseteq \mathbb{R}^{D}$ be the current set of points. The cardinality of $S$ is denoted by $n$. Our transformed data structure will be completely rebuilt after a sufficiently long sequence of updates. Let $S_{0}$ be the set of points at the moment of the most recent rebuilding and let $n_{0}$ be its size at that moment.

As in the previous section, for each $p \in \mathbb{R}^{D}$, box $(p)$ denotes the smallest box centered at $p$ that contains at least $(2 D+2)^{D}$ points of $S \backslash\{p\}$. The set of all points of $S \backslash\{p\}$ that are in the interior of this box is denoted by $N(p)$. If $p \in S_{0}$, then $\operatorname{box}_{0}(p)$ denotes the smallest box centered at $p$ that contains at least $(2 D+2)^{D}$ points of $S_{0} \backslash\{p\}$.

\section{The transformed closest pair data structure:}

1. The set $S$ is partitioned into sets $A$ and $B$ such that $A \subseteq\left\{p \in S: p \in S_{0} \wedge\right.$ $\left.\operatorname{box}(p) \subseteq \operatorname{box}_{0}(p)\right\}$.

2. The distances of the multiset $\{d(p, q): p \in A, q \in N(p)\}$ are stored in a heap. With each distance, we store the corresponding pair of points.

3. The boxes of the set $\left\{b o x_{0}(p): p \in S_{0}\right\}$ are stored in a list, called the box list. With each element $b_{0} x_{0}(p)$ in this list, we store a bit having value true if and only if $p \in A$. Moreover, if $p \in A$, we store with $b_{0} x_{0}(p)$ the box box $(p)$.

4. The boxes of the set $\left\{b o x_{0}(p): p \in S_{0}\right\}$ are stored in the static data structure of Theorem 5. This structure is called the box tree. With each box in this structure, we store a pointer to its occurrence in the box list.

5. The points of $S$ are stored in a dictionary. With each point $p$, we store a bit indicating whether $p$ belongs to $A$ or $B$. If $p \in A$, then we store with $p$

(a) a pointer to the occurrence of $b_{0 x}(p)$ in the box list, and

(b) a list containing the elements of $N(p)$. For convenience, we also call this list $N(p)$. With each point $q$ in $N(p)$, we store a pointer to the occurrence of $d(p, q)$ in the heap. 
6. The set $B$ is stored in the dynamic data structure $D S$. This structure is called the $B$-structure.

First we prove that this data structure indeed enables us to find a closest pair of the current set $S$ in $O(1)$ time.

Lemma 15 Let $\delta$ be the minimal distance stored in the heap and let $\delta^{\prime}$ be the distance of a closest pair in $B$. Then, $\min \left(\delta, \delta^{\prime}\right)$ is the distance of a closest pair in the set $S$.

Proof: Let $(P, Q)$ be a closest pair in $S$. We distinguish two cases.

Case 1: At least one of $P$ and $Q$ is contained in $A$.

Assume w.l.o.g. that $P \in A$. Since box $(P)$ contains at least $1+(2 D+2)^{D}$ points of $S$, it follows in the same way as in the proof of Lemma 14 that $Q$ is contained in the interior of this box. Hence, $Q \in N(P)$ and, therefore, the distance $d(P, Q)$ is stored in the heap. Clearly, the heap only contains distances of the current set $S$. Therefore, $\delta=d(P, Q)$. Moreover, since $d(P, Q)$ is the minimal distance in $S$, we have $d(P, Q) \leq \delta^{\prime}$. This proves that $d(P, Q)=\min \left(\delta, \delta^{\prime}\right)$.

Case 2: Both $P$ and $Q$ are contained in $B$.

Since $d(P, Q)$ is the minimal distance in $S$ and since the heap only stores distances between points of the current set $S$, we have $\delta^{\prime}=d(P, Q)$ and $d(P, Q) \leq \delta$. Therefore, $d(P, Q)=\min \left(\delta, \delta^{\prime}\right)$.

Initialization: At the moment of initialization, $S=S_{0}=A$ and $B=\emptyset$. Using Vaidya's algorithm [24], compute for each point $p$ in $S$ its $1+(2 D+2)^{D} L_{\infty^{-}}$ neighbors. The point among these neighbors having maximal $L_{\infty}$-distance to $p$ determines $\operatorname{box}(p)=b_{o x}(p)$. The neighbors (except $p$ itself) that are contained in the interior of this box form the list $N(p)$. It is clear how the rest of the data structure can be built. Note that each element $b_{0} x_{0}(p)$ in the box list has a bit with value true.

Now we can give the update algorithms. If a point $p$ of $S_{0}$ is deleted, it may be inserted again during some later update operation. If this happens, $p$ is assumed to be a new point, i.e., it is assumed that $p$ does not belong to $S_{0}$ again. In this way, an inserted point always belongs to $B$.

The insertion algorithm: Let $p \in \mathbb{R}^{D}$ be the point to be inserted. Assume w.l.o.g. that $p \notin S$.

1. Insert $p$ into the dictionary and store with $p$ a bit saying that $p$ belongs to $B$. Then, insert $p$ into the $B$-structure.

2. Using the box tree, find all boxes that contain $p$. For each reported element $b o x_{0}(q)$, follow the pointer to its occurrence in the box list. If the bit of box $(q)$ has value true, then check if $p$ is contained in the interior of box $(q)$. If so, do the following:

(a) Search in the dictionary for $q$. Insert $p$ into $N(q)$, insert $d(q, p)$ into the heap and store with $p$ a pointer to $d(q, p)$. 
(b) If $N(q)$ has size less than $(2 D+2)^{D}$, then the insertion algorithm is completed. Otherwise, let $r_{1}, \ldots, r_{l}$ be all points of $N(q)$ that are at maximal $L_{\infty}$-distance from $q$. For each $1 \leq i \leq l$, delete $r_{i}$ from $N(q)$ and delete $d\left(q, r_{i}\right)$ from the heap. Finally, replace box $(q)$ - which is stored with box $0(q)$ in the box list - by the box centered at $q$ having $r_{1}$ on its boundary, being the new box $(q)$.

It is easy to verify that this algorithm correctly maintains the data structure. Note that since $A \subseteq\left\{p \in S: p \in S_{0} \wedge\right.$ box $\left.(p) \subseteq \operatorname{box}_{0}(p)\right\}$, all boxes box $(q), q \in A$, that contain $p$ are found in Step 2.

The deletion algorithm: Let $p \in S$ be the point to be deleted.

1. Search for $p$ in the dictionary. If $p \in B$, then delete $p$ from this dictionary and from the $B$-structure.

Otherwise, if $p \in A$, follow the pointer to $\operatorname{box}_{0}(p)$ in the box list and set its bit to false. Moreover, for each point $q$ in $N(p)$, delete the distance $d(p, q)$ from the heap. Then, delete $p$ from the dictionary.

2. Using the box tree, find all boxes that contain $p$. For each reported element $b o x_{0}(q)$, follow the pointer to its occurrence in the box list. If the bit of box $(q)$ has value true, then check if $p$ is contained in box $(q)$. If so, do the following:

Set the bit of $b_{0} x_{0}(q)$ to false. Search in the dictionary for $q$. For each point $r$ in $N(q)$, delete $d(q, r)$ from the heap. Then, delete the pointer from $q$ to $b o x_{0}(q)$, delete the list $N(q)$, and store with $q$ a bit saying that it belongs to $B$. Finally, insert $q$ into the $B$-structure.

This concludes the description of the update algorithms. In order to guarantee a good space bound, we occasionally rebuild the data structure:

Rebuild: Recall that $n_{0}$ is the size of $S$ at the moment we initialize the structure. After $f\left(n_{0}\right)$ updates have been performed, we discard the entire structure and initialize a new data structure for the current $S$.

We analyze the complexity of the transformed data structure. First note that the initial and current sizes $n_{0}$ and $n$ are proportional: Since $f\left(n_{0}\right) \leq n_{0} / 2$, we have $n \leq n_{0}+f\left(n_{0}\right) \leq 3 n_{0} / 2$ and $n \geq n_{0}-f\left(n_{0}\right) \geq n_{0} / 2$.

The total size of the heap, the box list, the box tree and the dictionary is bounded by $O\left(n+n_{0}\right)=O(n)$. Consider the $B$-structure. Initially, this structure is empty. With each insertion, we insert one point into it, whereas with each deletion at most a constant number of points are inserted. (See Lemma 8 for the constant. Note that $c_{D}=(2 D+2)^{D}$.) Therefore, the $B$-structure stores $O\left(f\left(n_{0}\right)\right)=O(f(n))$ points and it has size $O(S(f(n)))$.

During each update operation, we perform a constant number of queries and updates in the various parts of the structure. Therefore, by Theorem $5, O\left((\log n)^{D-1}+\right.$ $U(f(n)))$ time is spent per update, in case the data structure is not rebuilt. 
Consider the initialization. We can compute for each point its $1+(2 D+2)^{D} L_{\infty^{-}}$ neighbors in $O\left(n_{0} \log n_{0}\right)$ time. (See [24].) By Theorem 5, the static box tree can also be built in $O\left(n_{0} \log n_{0}\right)$ time. It is clear that the rest of the data structure can be built within these time bounds. Hence, the entire initialization takes $O\left(n_{0} \log n_{0}\right)$ time. Since we do not rebuild during the next $f\left(n_{0}\right)$ updates, the initialization adds

$$
O\left(\frac{n_{0} \log n_{0}}{f\left(n_{0}\right)}\right)=O((n \log n) / f(n))
$$

to the overall amortized update time. This proves:

Theorem 7 Let $D S$ be any data structure for the dynamic closest pair problem. Let $S(n)$ and $U(n)$ denote the size and update time of $D S$, respectively. Let $1 \leq f(n) \leq n / 2$ be a non-decreasing integer function. Assume that $S(n), U(n)$ and $f(n)$ are smooth.

We can transform DS into another data structure for the dynamic closest pair problem having

1. size $O(n+S(f(n)))$, and

2. an amortized update time of $O\left((\log n)^{D-1}+U(f(n))+(n \log n) / f(n)\right)$.

Corollary 2 Let $S$ be a set of $n$ points in $\mathbb{R}^{D}, D \geq 3$, and let $1 \leq t \leq \infty$. There exists a data structure of size $O(n)$ that maintains an $L_{t}$-closest pair of $S$ in $O\left((\log n)^{D-1} \log \log n\right)$ amortized time per insertion and deletion.

Proof: We apply Theorem 7 twice. Let $D S$ be the data structure of Theorem 6, i.e., $S(n)=O\left(n(\log n)^{D-1}\right)$ and $U(n)=O\left((\log n)^{D-1} \log \log n\right)$. Moreover, let $f(n)=$ $n /\left((\log n)^{D-2} \log \log n\right)$. Then, Theorem 7 gives a closest pair structure $D S^{\prime}$ of size $S^{\prime}(n)=O(n \log n / \log \log n)$ having $U^{\prime}(n)=O\left((\log n)^{D-1} \log \log n\right)$ amortized update time. Applying Theorem 7 to $D S^{\prime}$ with $f^{\prime}(n)=n \log \log n / \log n$ proves the corollary.

Corollary 3 Let $S$ be a set of $n$ points in the plane and let $1 \leq t \leq \infty$. For any non-negative integer constant $k$, there exists a data structure

1. of size $O\left(n \log n /(\log \log n)^{k}\right)$ that maintains an $L_{t}$-closest pair of $S$ at a cost of $O(\log n \log \log n)$ amortized time per insertion and deletion,

2. of size $O(n)$ that maintains an $L_{t}$-closest pair of $S$ in $O\left((\log n)^{2} /(\log \log n)^{k}\right)$ amortized time per insertion and deletion.

Proof: The proof is by induction on $k$. For $k=0$, the result claimed in 1 . follows from Theorem 6. Let $k \geq 0$ and let $D S$ be a closest pair data structure having size $S(n)=O\left(n \log n /(\log \log n)^{k}\right)$ and update time $U(n)=O(\log n \log \log n)$. Applying Theorem 7 with $f(n)=n(\log \log n)^{k} / \log n$ gives a closest pair structure of size $O(n)$ having $O\left((\log n)^{2} /(\log \log n)^{k}\right)$ amortized update time.

On the other hand, applying Theorem 7 to $D S$ with $f(n)=n / \log \log n$ gives a closest pair structure of size $O\left(n \log n /(\log \log n)^{k+1}\right)$ with an amortized update time of $O(\log n \log \log n)$. 


\section{Concluding remarks}

We have given new techniques for solving the dynamic approximate nearest-neighbor problem and the dynamic closest pair problem. Note that for the static version of the first problem an optimal solution-having logarithmic query time and having linear size - is known. (See [2].) It would be interesting to solve the dynamic problem within the same complexity bounds and with a logarithmic update time.

For the dynamic closest pair problem, we obtained several new results. We first gave a data structure that improved the best structures that were known. Then we applied a general transformation to improve this solution even further. Note that if we apply this transformation several times, as we did, then we maintain a hierarchy of data structures similar to the logarithmic method for decomposable searching problems. (See [3].) It would be interesting to know if the ideas of this transformation can be applied to other problems. Finally, we leave as an open problem to decide whether there is an $O(n)$ space data structure that maintains the closest pair in $O(\log n)$ time per insertion and deletion.

\section{References}

[1] S. Arya and D.M. Mount. Approximate nearest neighbor queries in fixed dimensions. Proc. 4th Annual ACM-SIAM Symp. on Discrete Algorithms, 1993, pp. 271-280.

[2] S. Arya, D.M. Mount, N.S. Netanyahu, R. Silverman, A. Wu. An optimal algorithm for approximate nearest neighbor searching. Proc. 5th Annual ACM-SIAM Symp. on Discrete Algorithms, 1994, pp. 573-582.

[3] J.L. Bentley. Decomposable searching problems. Inform. Proc. Lett. 8 (1979), pp. $244-251$.

[4] J.L. Bentley and M.I. Shamos. Divide-and-conquer in multidimensional space. Proc. 8th Annual ACM Symp. Theory of Computing, 1976, pp. 220-230.

[5] M. Bern. Approximate closest-point queries in high dimensions. Inform. Proc. Lett. 45 (1993), pp. 95-99.

[6] B. Chazelle and L.J. Guibas. Fractional cascading I: A data structuring technique. Algorithmica 1 (1986), pp. 133-162.

[7] K.L. Clarkson. A randomized algorithm for closest-point queries. SIAM J. Comput. 17 (1988), pp. 830-847.

[8] H. Edelsbrunner, G. Haring and D. Hilbert. Rectangular point location in $d$ dimensions with applications. The Computer Journal 29 (1986), pp. 76-82.

[9] M. Golin, R. Raman, C. Schwarz and M. Smid. Randomized data structures for the dynamic closest-pair problem. Proc. 4th Annual ACM-SIAM Symp. on Discrete Algorithms, 1993, pp. 301-310. 
[10] M. Golin, R. Raman, C. Schwarz and M. Smid. Simple randomized algorithms for closest pair problems. Proc. 5th Canadian Conf. Computational Geometry, 1993, pp. 246-251.

[11] D.T. Lee. Two-dimensional Voronoi diagrams in the $L_{p}$-metric. J. ACM 27 (1980), pp. 604-618.

[12] G.S. Lueker. A data structure for orthogonal range queries. Proc. 19th Annual IEEE Symp. Foundations of Computer Science, 1978, pp. 28-34.

[13] K. Mehlhorn. Data Structures and Algorithms, Volume 3: Multi-Dimensional Searching and Computational Geometry. Springer-Verlag, Berlin. 1984.

[14] K. Mehlhorn and S. Näher. Dynamic fractional cascading. Algorithmica 5 (1990), pp. 215-241.

[15] F.P. Preparata and M.I. Shamos. Computational Geometry, an Introduction. Springer-Verlag, New York, 1985.

[16] J.S. Salowe. Enumerating interdistances in space. International Journal of Computational Geometry \& Applications 2 (1992), pp. 49-59.

[17] C. Schwarz. Data structures and algorithms for the dynamic closest pair problem. Ph.D. Thesis. Universität des Saarlandes, Saarbrücken, 1993.

[18] C. Schwarz, M. Smid and J. Snoeyink. An optimal algorithm for the on-line closest pair problem. Proc. 8th ACM Symp. on Computational Geometry, 1992, pp. 330336.

[19] M.I. Shamos and D. Hoey. Closest-point problems. Proc. 16th Annual IEEE Symp. Foundations of Computer Science, 1975, pp. 151-162.

[20] M. Smid. Maintaining the minimal distance of a point set in less than linear time. Algorithms Review 2 (1991), pp. 33-44.

[21] M. Smid. Rectangular point location and the dynamic closest pair problem. Proc. 2nd Annual International Symp. on Algorithms, Lecture Notes in Computer Science, Vol. 557, Springer-Verlag, Berlin, 1991, pp. 364-374. Full version to appear in Information and Computation.

[22] M. Smid. Maintaining the minimal distance of a point set in polylogarithmic time. Discrete Comput. Geom. 7 (1992), pp. 415-431.

[23] K.J. Supowit. New techniques for some dynamic closest-point and farthest-point problems. Proc. 1st Annual ACM-SIAM Symp. on Discrete Algorithms, 1990, pp. 84-90.

[24] P.M. Vaidya. An $O(n \log n)$ algorithm for the all-nearest-neighbors problem. Discrete Comput. Geom. 4 (1989), pp. 101-115. 
[25] D.E. Willard and G.S. Lueker. Adding range restriction capability to dynamic data structures. J. ACM 32 (1985), pp. 597-617.

[26] A.C. Yao. On constructing minimum spanning trees in $k$-dimensional spaces and related problems. SIAM J. Comput. 11 (1982), pp. 721-736. 